\title{
Highly flexible and non-toxic natural polymer gel electrolyte for printed supercapacitors for loT
}

\author{
Anna Railanmaa ${ }^{1}$ (D) $\cdot$ Manu Kujala $^{1} \cdot$ Jari Keskinen $^{1} \cdot$ Terho Kololuoma $^{2} \cdot$ Donald Lupo $^{1}$
}

Received: 4 December 2018 / Accepted: 30 January 2019 / Published online: 5 February 2019

(c) The Author(s) 2019

\begin{abstract}
Gelatin offers a safe and sustainable alternative for gel electrolytes in printed supercapacitors. In this work, the performance of a non-toxic natural polymer electrolyte is studied in screen and stencil-printed supercapacitors. The basic electrical properties are determined with samples prepared on laboratory scale; subsequently, roll-to-roll printed samples are used to observe behavior of the samples under severe mechanical stress. With a $2 \mathrm{M} \mathrm{NaCl}$ gel electrolyte, electrical performance equal to that of a $1 \mathrm{M}$ liquid electrolyte is reached, and the devices endure bending down to a $10 \mathrm{~mm}$ radius. Even below that, functional devices are successfully measured, although leakage current levels deteriorate somewhat during bending. However, even these samples recover to baseline levels after they are straightened and can be thus used in various applications, since devices with no short circuits or permanent degradation can be successfully prepared.
\end{abstract}

\section{Introduction}

Supercapacitors $[1,2]$ are a versatile energy storage option in various applications thanks to their energy storage mechanism by electrical double layer, which increases cycle life and allows the use of safer ionic conductors and electrode materials than in traditional batteries. The applications can be stationary, but in many cases, for example in wearable or otherwise portable devices, the supercapacitors will be subjected to various amounts of deformation.

Supercapacitors can be utilized in sensor and actuator systems for Internet of Things (IoT) to provide temporary power storage when energy from the environment is not continually available. For example, supercapacitors can be used together with photovoltaic cells in autonomous sensor networks and IoT applications to store energy and power the system in the dark $[3,4]$. Other potential applications

Electronic supplementary material The online version of this article (https://doi.org/10.1007/s00339-019-2461-8) contains supplementary material, which is available to authorized users.

Anna Railanmaa

anna.railanmaa@tuni.fi

1 Information Technology and Communication Sciences, Tampere University, 33720 Tampere, Finland

2 VTT Technical Research Centre of Finland Ltd, Kaitoväylä 1, 90571 Oulu, Finland include, e.g., monitoring temperature for food safety, or storing energy from motion in wearable devices for interfaces such as displays or low-power data transmission [5]. It is important that alternative material options for these applications are studied to ensure user safety as well as environmental sustainability.

One potential improvement is replacement of the liquid electrolyte with a solid alternative prepared from non-toxic, non-corrosive and renewable materials. This will improve end-user safety significantly by greatly reducing the risk of leakage and making the devices less problematic to dispose of and recycle. Moreover, even if leakage would occur, the safety risks to the user would be eliminated with benign materials.

One of the key properties of gel electrolytes is their ability to withstand significant mechanical stress without allowing contact between the electrodes, while simultaneously maintaining an internal structure that allows sufficient ion mobility within the electrolyte. This also reduces the number of components required in assembling the supercapacitors, as the functionality of two components, the electrolyte and the separator, is combined.

Hydrogels offer an interesting opportunity to modify traditional aqueous electrolytes into a form in which leakage problems are reduced and the need for an additional separator layer is removed, but the good ion mobility is still maintained by the structure consisting mostly of water [6]. Hydrogels can be manufactured from a variety 
of source polymers, both renewable and non-renewable in nature [7].

The source polymer should have good hydrophilicity to accommodate the maximum amount of water in the structure, and especially for wearables and, e.g., medical or foodstuff-related applications, must be non-toxic and noncorrosive. This makes certain natural polymers like gelatin, starch, pectin, and agar very appealing alternatives, as they are hydrophilic but also environmentally safe, renewable, and non-toxic [8-13]. Many materials used in research of flexible supercapacitors pose a potential threat to the environment and the health of the end user: ionic liquids, nanomaterials, and corrosive electrolytes are all more or less problematic in terms of disposal and/or user safety [14-17]. Investigations into flexible supercapacitors with aqueous, neutral electrolytes are scarce, even though they are essential for wearables and user safety.

The potential of printed supercapacitors has been already demonstrated as an integrated part of energyautonomous electronic devices [5, 18]. One major advantage of printed devices is their potentially low cost, which makes them ideal for distributed electronics. In many potential IoT applications, low-power circuitry will be critical, since the energy available for harvesting will be limited. For such applications the key motivations for supercapacitors, in contrast to those for common commercial applications, are high cycle life, low cost, and disposability rather than extremely high power density.

In this work, we present the properties of a gelatin gel electrolyte in printed supercapacitors and study both the electrical and mechanical behaviors of the devices. The focus here is on the performance of the gel. Thus, the other architectural characteristics of the supercapacitors were chosen based on work previously reported by Kraft et al. [19], which compared the performance of various combinations of substrates and sealing methods. The substrate and the sealing method for this work were chosen so that the effect of these additional structural components on the failure rate of the supercapacitors under mechanical stress was minimal. Moreover, the structure is composed of safe, inexpensive and easily disposable materials, with carbonbased electrodes and current collectors.

We report the electrical performance of the gel electrolyte when it is integrated into a functional, carbon-based printed supercapacitor produced both on laboratory scale by hand and by roll-to-roll printing. The gel electrolyte has previously been studied independently for its ionic conductivity as a part of another publication [6]. The performance of the gel electrolyte is comparable to an aqueous liquid electrolyte with the same salt as the ionic conductor in terms of the major characteristics: capacitance, equivalent series resistance, and leakage current.

\section{Experimental procedure}

The gel electrolyte used in this work was chosen based on the characterization of various gel compositions and salt concentrations studied in Railanmaa, 2017 [6]. The $2 \mathrm{~mol} \mathrm{~L}^{-1}$ gelatin gel used in this study combines good ionic conductivity, comparable to the previously studied $1 \mathrm{~mol} \mathrm{~L}^{-1} \mathrm{NaCl}$ (aq) [20], and the sufficient mechanical strength observed in initial testing. The gel was prepared using gelatin powder from porcine skin (Sigma-Aldrich) and sodium chloride (Sigma-Aldrich) dissolved in deionized water. First, the $\mathrm{NaCl}$ was dissolved in the water to produce a $2 \mathrm{~mol} \mathrm{~L}^{-1}$ solution, to which the gelatin was then dissolved by briefly bringing the solution to a boil. The glycerol plasticizer was mixed in before cooling the solution, after which the gel was allowed to set in room temperature.

Initially, supercapacitors were prepared by hand by stencil printing to obtain information about specific capacitance as well as other electrical properties. The key properties of the supercapacitors were studied with the handprinted samples, but for a further study into the effects of mechanical stress, a batch of roll-to-roll screen-printed current collectors and electrodes was prepared on a pilot printing line on a larger scale to produce the required large quantity of samples.

The current collectors and electrodes for the initial study of the electrical characteristics of the gel supercapacitors were produced by stencil printing ( $120 \mu \mathrm{m}$ stainless steel stencils) on PET film (Melinex ST506 from DuPont Teijin Films). Henkel Electrodag PF407C graphite ink was used to print the $20 \mathrm{~mm} \times 30 \mathrm{~mm}$ current collectors, which were dried at $120^{\circ} \mathrm{C}$ for $15 \mathrm{~min}$. For the electrodes, an in-house activated carbon (AC) ink was prepared. $1.7 \mathrm{~g}$ of low viscosity chitosan (from shrimp shell, Sigma-Aldrich) was dissolved overnight in $70 \mathrm{~g}$ deionized water and $0.7 \mathrm{~g}$ acetic acid. $30.9 \mathrm{~g}$ of activated carbon powder (Kuraray YP-80F) was mixed into the solution and approx. $15 \mathrm{ml}$ of additional water was mixed in to produce a paste with a stencil-printable viscosity. Electrodes of $18 \mathrm{~mm} \times 10 \mathrm{~mm}$ were printed with the AC paste on top of one end of the current collector and allowed to air dry at room temperature before assembling. The samples were weighed before and after printing the $\mathrm{AC}$ ink, to determine the mass of the active material. The total weight of the dry $\mathrm{AC}$ ink was used for determining the specific capacitance of the supercapacitors, including the weight of the binder.

The electrolyte gel was melted on a hot plate at $65{ }^{\circ} \mathrm{C}$ and dispensed onto the electrodes and over the edges of the current collectors with a Pasteur pipette. An excess of gel was used to allow the air in the pores of the AC ink to bubble to the surface, from where the bubbles could be 
selectively pipetted off the sample. After this, the samples were topped with a small amount of the gel (1 drop for thin layers, 3-4 for thicker) to provide the needed separation between the electrodes once assembled, and allowed to set in ambient air for approximately $10 \mathrm{~min}$. Then, the supercapacitors were assembled in a head-on configuration, using a frame of $3 \mathrm{M} 200 \mathrm{MP} / 468 \mathrm{MP}$ adhesive film, and the gelatin was allowed to set further and diffuse together overnight before electrical characterization.

The architecture for the bending study was chosen based on earlier results comparing substrates and sealing methods and their performance under mechanical stress [19]. The current collectors and electrodes for the bending study samples were mass manufactured by rotary screen printing on a multi-layer polymer film, with a PP/PA/EVOH/PA/PE structure. The current collectors and electrodes were printed on the PE side. Roll-to-roll prints were conducted in co-operation with VTT Technical Research Centre of Finland, where the current collectors were printed at the ROKO facility with Henkel Electrodag PF407C graphite ink at line speed $2 \mathrm{~m}$ $\min ^{-1}$, and cured at $90{ }^{\circ} \mathrm{C}$ for $2 \mathrm{~min}$. The same activated carbon ink that was used for stencil printing the electrodes for the initial study was used for the roll-to-roll printing at line speed $2 \mathrm{~m} \mathrm{~min}^{-1}$, and dried at $90{ }^{\circ} \mathrm{C}$ for $2 \mathrm{~min}$. The electrolyte gel was deposited by pipetting the molten solution on top of both electrodes and was allowed to set in room temperature to a solid state before assembling the supercapacitor and sealing the device with the adhesive $3 \mathrm{M}$ film.

The electrical characterization protocol was the same for both the initial samples and the bending samples. The initial samples were characterized with a BioLogic BCS-805 battery cycling system and the bending samples were characterized with a Maccor 4300 workstation (Maccor Inc., USA), both programmed to follow an identical measurement protocol in accordance with IEC standard 62391-1:2006 [21]. The measurement was conducted at three different measurement currents: 1, 3 and $10 \mathrm{~mA}$. Each current was used to first cycle the supercapacitor from 0 to $0.9 \mathrm{~V}$ and back three times, then charge it to 0.9 and hold for $30 \mathrm{~min}$, discharge to $0 \mathrm{~V}$ and charge back to $0.9 \mathrm{~V}$ for a $1 \mathrm{~h}$ hold to define leakage current, then discharge back to $0 \mathrm{~V}$ again. Finally, after measurements at each current, cyclovoltammograms of the supercapacitor were measured for four cycles at 100 , 50,10 and $5 \mathrm{mV} \mathrm{s}^{-1}$ each. Specific capacitance values were calculated from the ratio of supercapacitor capacitance to the total electrode mass of two electrodes.

Electrical properties of the initial samples were measured only once to determine the original device characteristics. The bending samples and their corresponding reference samples were measured three times each: before deformation, during static bending and in a straightened state after being bent. The reference samples were identical in structure to the bending samples, i.e., they contained the gel electrolyte as well. Reference samples were measured an equal number of times at equal intervals without mechanical deformation to provide a baseline for the aging of the devices, since especially capacitance and leakage current are somewhat dependent on the number of measurement cycles experienced by the supercapacitor [20]. Because of this, there is unavoidably some small differences between the before and after values, regardless of the bending.

For the bent samples, the bending radii were $37.5,25$, $17.5,10$, and $5 \mathrm{~mm}$. After receiving the results from the first tests on the smallest bending radius of $5 \mathrm{~mm}$, another measurement round with a thicker gel layer was executed to evaluate if a thicker separator layer would protect against the increasing leakage current rates.

The internal structure of the supercapacitors was studied with scanning electron microscopy (SEM). All the supercapacitors were assembled and electrically characterized first, to ensure that only working supercapacitors were imaged. After this, the samples were cut open using a fresh scalpel and horizontal motion to minimize any deformation to the gel and prevent the top electrode from pushing down on the sample.

\section{Results and discussion}

It was hypothesized that the thickness of the gel electrolyte might affect some of the electrical characteristics of the supercapacitor, especially the ESR, and thus the performance of different thicknesses of gelatin layers was compared. The approximate thicknesses of the gel separator layers were obtained from cross-sectional SEM images of the assembled and previously electrically characterized supercapacitors.

In Fig. 1a, the cross-section of a thinner layer of gelatin is presented. The layered structure of the devices is well visible, starting from the PET substrate at the top, then current collector, AC electrode, gelatin electrolyte, and then an identical structure mirrored at the bottom. The gelatin layer is approximately $20 \mu \mathrm{m}$ thick and appears uniform, and there is no indication of a junction in the middle of the gelatin layer from the assembly. Instead, the two layers have diffused together seamlessly.

The supercapacitor in Fig. 1b has a thicker gelatin layer but otherwise identical structure with the sample in Fig. 1a. The thicker gel layers have merged seamlessly as well, and the gelatin structure appears continuous. As can be seen in the following closer inspection of the electrical and mechanical properties, the thinner gel layer is already able to withstand significant deformation without serious harm to the electrical performance. Therefore, the thicker layers were not investigated further after the initial tests, except for the smallest bending radius. However, in use cases where severe 
Fig. 1 SEM image of a thinner (a) and a thicker (b) gelatin gel separator. Both layer thicknesses were sufficient in preventing short circuits during significant bending
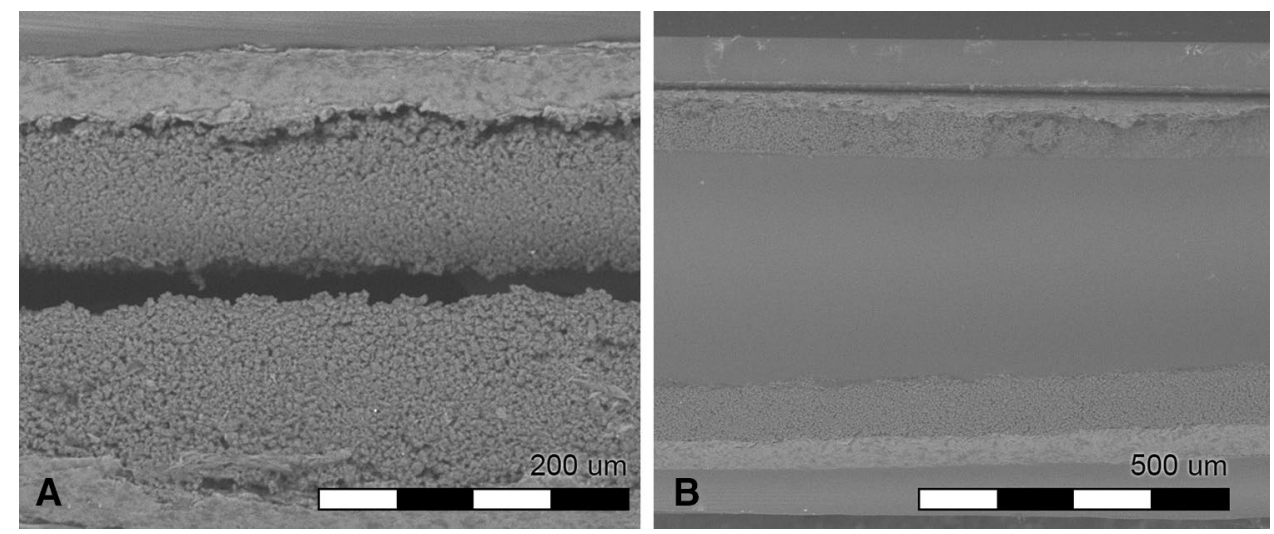

Table 1 The specific capacitance, ESR and leakage current values for samples with thicker and thinner gelatin gel separators

\begin{tabular}{llll}
\hline Sample type & $\begin{array}{l}\text { Specific C }(\mathrm{F} \\
\left.\mathrm{g}^{-1}\right)\end{array}$ & Leakage $(\mathrm{uA})$ & ESR $(\Omega)$ \\
\hline Thin gel & 24.6 & 8.5 & 25.4 \\
& 26.3 & 7.6 & 27.4 \\
& 26.4 & 5.8 & 26.6 \\
& 25.6 & 6.5 & 31.5 \\
Average & 27.0 & 5.9 & 30.8 \\
Thick gel & 26.0 & 6.9 & 28.4 \\
& 23.4 & 8.7 & 27.0 \\
& 22.8 & 7.2 & 31.0 \\
& 23.5 & 8.8 & 26.3 \\
& 23.2 & 9.9 & 27.0 \\
& 23.2 & 7.1 & 26.0 \\
& 21.7 & 7.5 & 30.5 \\
Average & 18.6 & 6.4 & 23.3 \\
\hline
\end{tabular}

bending is inevitable and dependability is critical, the gel layer can be moderately thickened without increasing leakage current or ESR values unreasonably.

Hand-printed samples were used to determine the specific capacitance values. Specific capacitance cannot be obtained for the roll-to-roll printed samples due to the manufacturing technique, but the area of the electrodes is the same with both manufacturing methods, so the absolute values per device can be directly compared, even though variation in layer thickness causes some variation in the absolute capacitance. All other component dimensions between the hand made and roll-to-roll printed samples were identical as well.

In Table 1, the specific capacitance values, ESR and leakage current levels are presented for thicker and thinner gel layers in stencil-printed supercapacitors. In comparison to previous studies [20, 22], the ESR level is slightly elevated, which can be mainly explained by the different drying conditions. In this study, no forced convection was used to dry the hand-printed samples to make the results as comparable to the roll-to-roll printed samples as possible, since no forced convection is available on the pilot line used for the upscaling. However, it should be noted that in lowpower autonomous devices the requirements in regards to ESR are very different from current mainstream applications like electric vehicles, and this level of ESR should not pose an issue. The difference in specific capacitance between the thicker and thinner samples is within the normal variation between individual samples. Leakage current appears to suffer a minor increase as a result of the increasing layer thickness of the gel, probably because the quantity of impurities is increased, but the change is negligible in comparison to the relative change in layer thickness.

The results of the bending study (Figs. 2, 3, 4, 5, 6, 7) demonstrate that the supercapacitors without an additional separator component are able to withstand notable amounts of deformation without major deterioration of electrical properties. The base line change, caused by aging of the supercapacitors during the charge/discharge cycles in characterization, can be seen with the reference samples, which have been measured an equal amount of times but without the mechanical stress from bending. By comparing the bent samples to this baseline performance, the effect of the mechanical stress can be evaluated. The changes are presented as percentiles to minimize the effect of sample-tosample variation in absolute values. The absolute measured values for the samples can be found in the supplementary information in Figures S1-S6 and the standard deviations between all the samples are presented in Table S1.

At the first measurement round, the capacitance values per device are similar to the samples prepared by hand, which indicates that upscaling the manufacturing process can be done easily and reliably. Other key electrical characteristics, ESR and leakage current, are similar as well. The effect of bending is manifested during the second measurement round, where the supercapacitors are measured while statically bent. During this time, the reference samples 


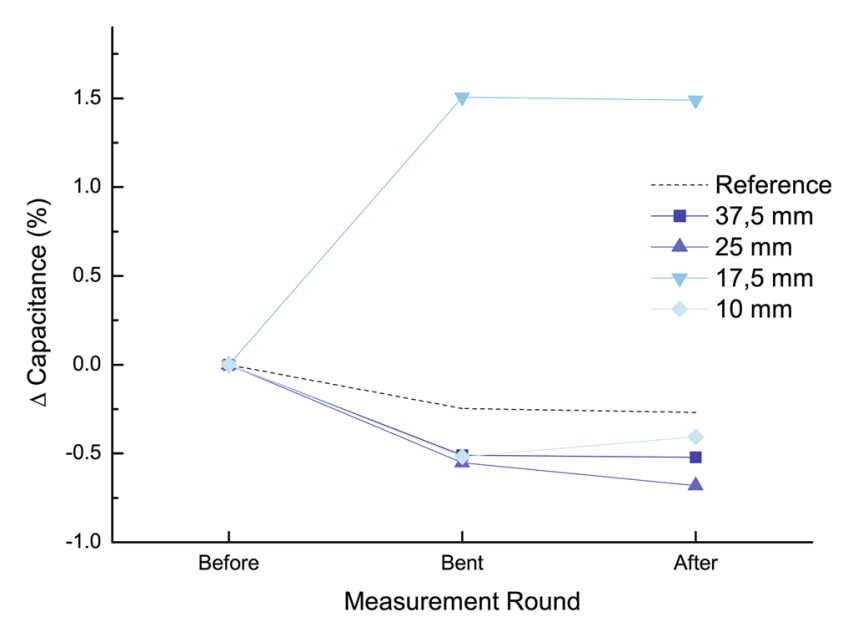

Fig. 2 Change in capacitance at the largest radius with the unbent reference

remained unbent but in the figures they have been marked to the same scale at the $\mathrm{x}$-axis to provide a direct point of comparison. The largest radius has hardly any noticeable difference to the reference samples, considering sample-tosample variance, and it appears that the gel electrolyte is well able to prevent any short circuits between electrodes. For the most part, the samples also recover close to the baseline level of the reference samples once the static stress is removed and no residual effect can be observed afterwards. Some individual samples did not survive the entire measurement sequence, including one of the references, but this is mainly due to the general yield of the manual assembly process and sample-to-sample variation could be reduced through higher automation level in manufacturing.

The recovery to baseline values can be explained by the performance of the gel electrolyte itself, since earlier studies with liquid electrolyte have yielded results indicating that the other components of the device produce very small changes in electrical performance [19]. As seen in the cross sectional SEM images of the combined gel layers inside the supercapacitors (Fig. 1), the gel layers have merged seamlessly after assembly, indicating good self-healing properties in the gel layer. Additionally, when handling the gel as a selfstanding film, it is evident that during compression the gel is quite elastic and recovers back to original form when the stress is released. Effectively, the stress caused by bending is compression at the inner part of the electrolyte and tensile at the outer side, when wrapped around a cylindrical object. The tensile stress may cause some micro cracking in the top of the electrolyte layer and compression affects the layer thickness, bringing the electrodes closer together.

However, based on the described observations concerning the mechanical behavior of the gel, both the compression and cracking can be expected to recover as the stress

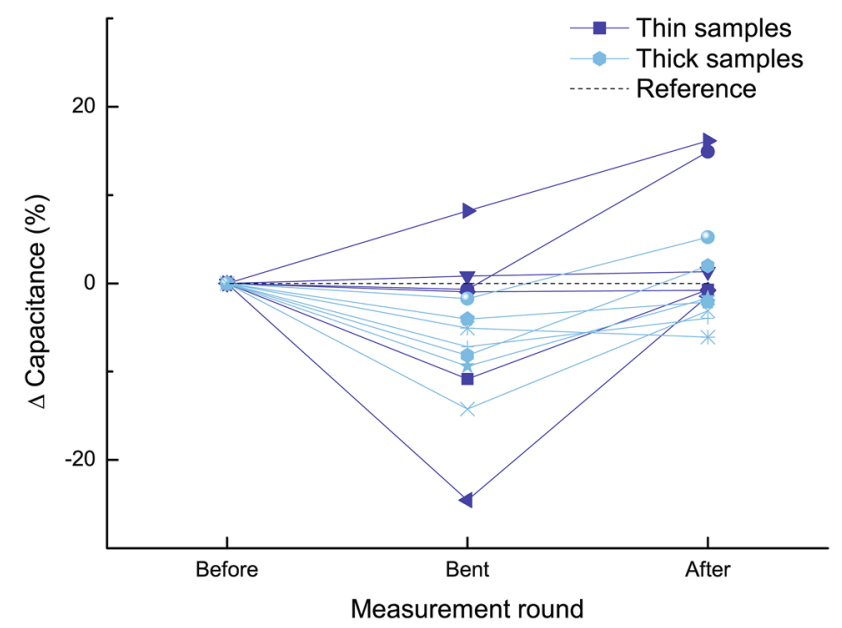

Fig. 3 Change in capacitance at the smallest bending radius for samples with thicker and thinner gel layers and the unbent reference

is released and device geometry is returned to its original form, resulting in recovery of electrical performance as well.

At the larger radii, the samples behave quite uniformly and the data are presented as averages for improved legibility for capacitance, ESR and leakage current in Figs. 2, 4 and 6 , respectively. The samples at the smallest radius of $5 \mathrm{~mm}$ have the largest sample-to-sample variance, especially during bending, and they are presented individually in Figs. 3, 5 , and 7 for capacitance, ESR and leakage current, respectively. The reference levels have been included in both cases for comparison. ESR for the $10 \mathrm{~mm}$ radius appears to differ somewhat from the larger radii, but this is mostly caused by a pair of individual samples in the series, which have slightly higher values. The separated values for each of the $10 \mathrm{~mm}$ samples have been presented in Figures S7-S9. Most of the samples even at this radius follow the baseline very well, but naturally harsher conditions will highlight the differences between samples, such as slight differences in packaging. The results for the $10 \mathrm{~mm}$ samples exhibit this phenomenon already to some extent and $5 \mathrm{~mm}$ samples show it even more pronouncedly.

In terms of capacitance, the $17.5 \mathrm{~mm}$ radius appears to differ from the others, but the difference observed here is within experimental uncertainty. Regarding the bending radii from 37.5 to $10 \mathrm{~mm}$, the leakage current appears to remain at an acceptable level during bending and the samples recover well from the bending as indicated by the after measurement.

The most obvious effect regarding the leakage current is observed on the smallest bending radius of $5 \mathrm{~mm}$, where the leakage current increases dramatically during bending for most samples. However, almost all of these samples recover to an acceptable level of leakage current after being returned to a straightened state and allowed to relax overnight, before 


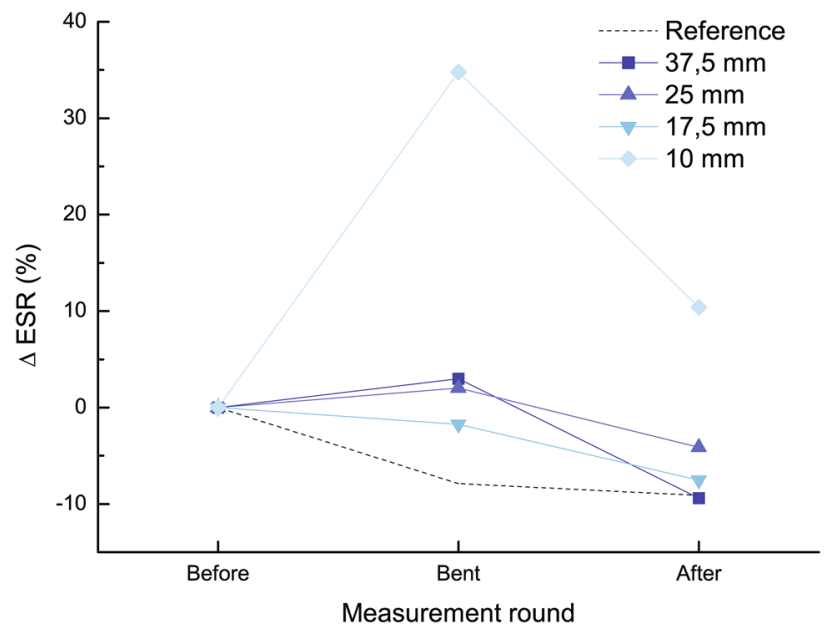

Fig. 4 Change in ESR at the largest radii with the unbent reference

the measurement on the straightened sample after bending. This behavior is curious, since it would be expected that in the occurrence of an Ohmic leak through the gelatin separator [1], the electrode-to-electrode connection would rapidly cause the measurement device to automatically abort the measurement. This was the case with one of the samples at the lowest bending radius, where the measurement during bending was interrupted by a safety feature. A severe enough short circuit through the gel to cause this kind of disruption requires only a very local puncture to the separator material, so it does not seem plausible that the measurable increase in leakage current presented in Fig. 7a would be the end result of different sizes of short circuits.

Instead, it would appear that the effect is caused by local compression of the gel, which brings the two electrodes closer together. Since a major source of leakage current in the supercapacitors arises from the impurities of the materials, limited by diffusion, reducing the distance between the electrodes increases the leakage level. Some impurities are able to shuttle between the electrodes in reduced and oxidized forms [1], and since the distance for these impurities to diffuse over is reduced, the effect of the shuttling is increased, increasing the total leakage current. As the distance between the electrodes is returned to normal after straightening the sample, the shuttle rate and thus the leakage current is returned to a normal level when compared with other samples and their aging behavior.

In the magnified image of Fig. 7b, it can be seen that some samples retain an acceptable level of leakage current even at this extreme bending radius, and the development from measurement to measurement equals that of the unbent reference samples. (In Fig. 7b, only the samples with decreasing leakage current from Fig. $7 \mathrm{a}$ are presented for legibility of the curves). This indicates that samples can be manufactured to withstand even this extreme level of

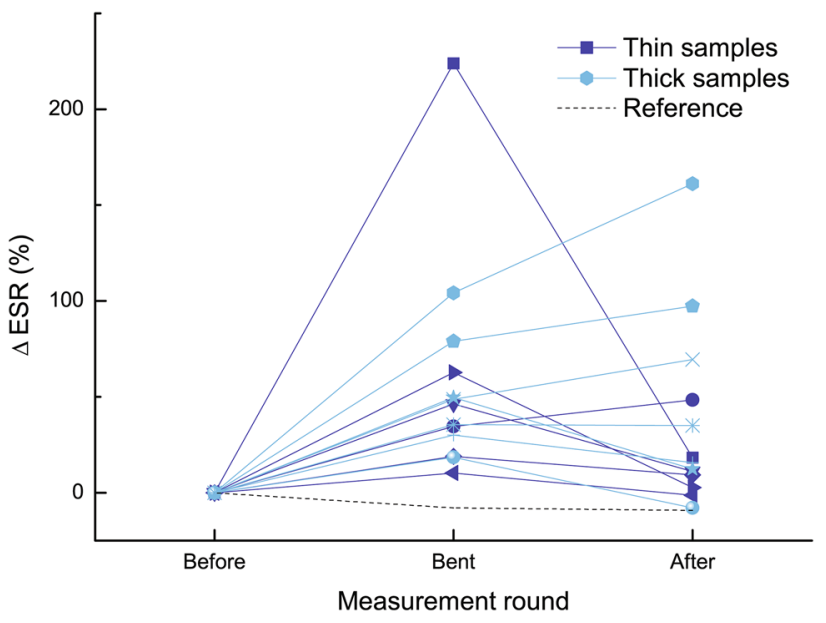

Fig. 5 Change in ESR at the smallest bending radius for samples with thicker and thinner gel layers and the unbent reference

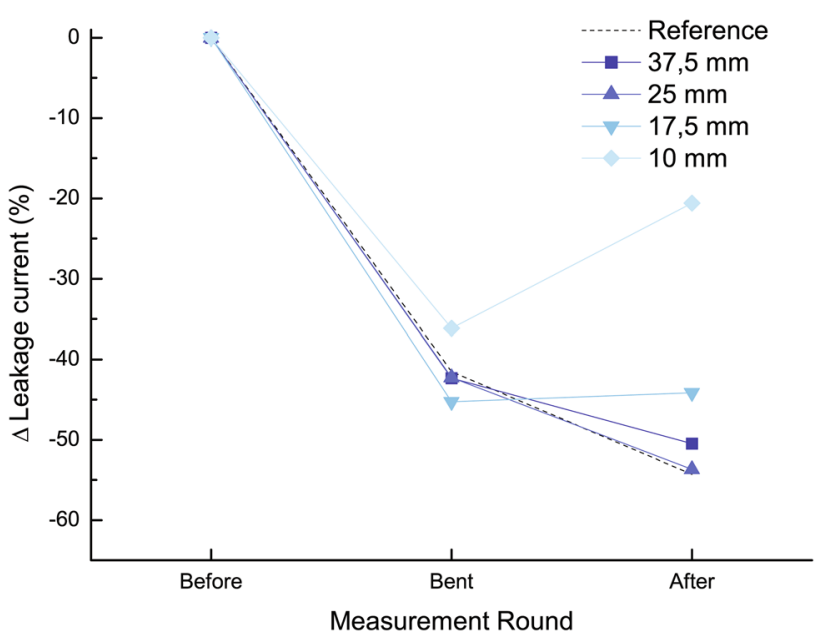

Fig. 6 Change in leakage currents at the largest radii with the unbent reference

mechanical stress. Naturally in samples prepared by hand in a laboratory, the deviation between samples is greater than in more automated systems and yield of the process can be improved in the future by process optimization.

\section{Conclusions}

Printed supercapacitors with a non-toxic, sustainable, aqueous gel electrolyte based on gelatin were subjected to static bending stress from 37.5 to $5 \mathrm{~mm}$ radius and characterized for electrical performance.

Supercapacitors comprising this electrolyte show a good specific capacitance level of approx. 20-25 $\mathrm{F} \mathrm{g}^{-1}$. Most leakage current levels are well below $10 \mu \mathrm{A}$ already at the first 

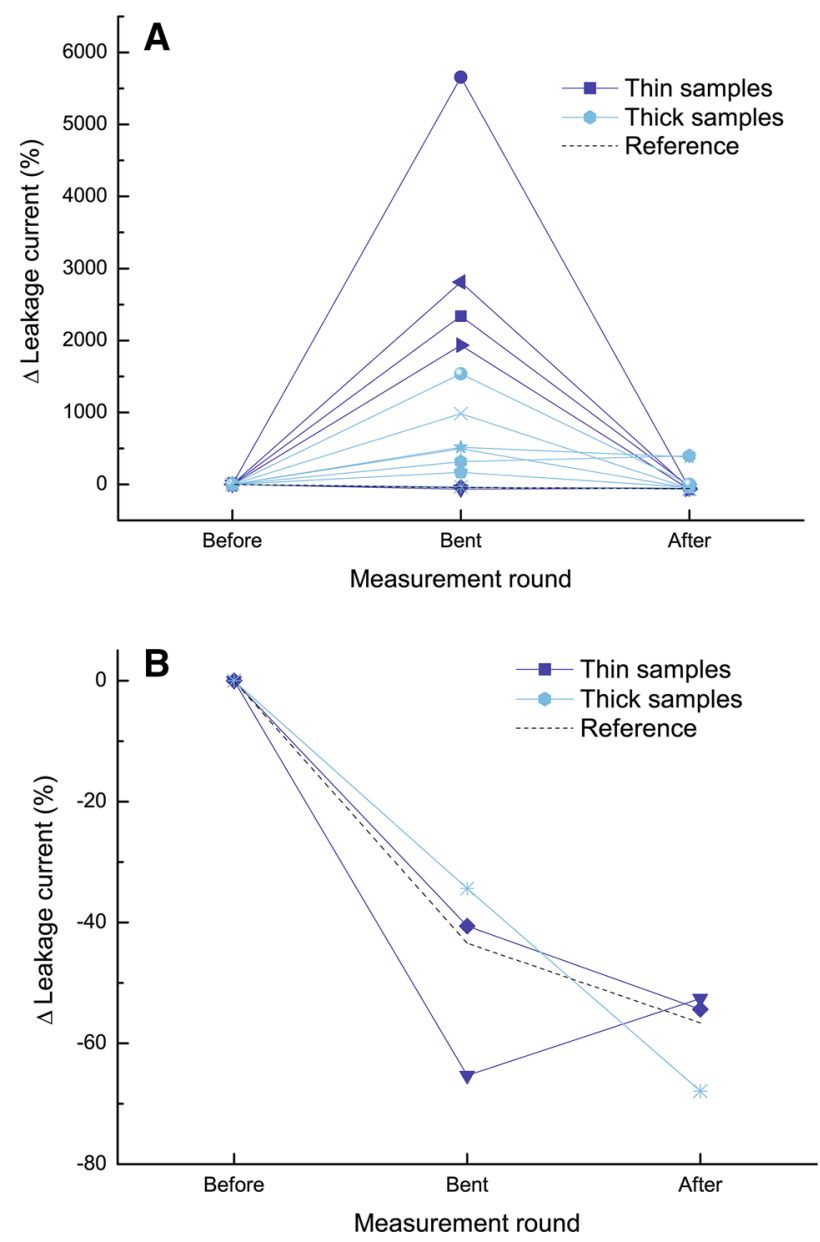

Fig. 7 Change in leakage current at the smallest bending radius for samples with thicker and thinner gel layers and the unbent reference. All samples in a and magnified view of selected samples in $\mathbf{b}$

measurement and decrease further during aging. Overall, the samples survived well under even very harsh bending conditions and recovered close to the reference level after bending. None of the manufactured gel supercapacitors exhibited physical leakage of the electrolyte, which is characteristic to a liquid electrolyte supercapacitor if the sealing of the package breaks due to mechanical stress. This improves end-user safety significantly and makes the devices more reliable in practical scenarios.

Acknowledgements The authors are indebted to the Finnish Funding Agency for Technology and Innovation [Dec. No. 40337/14] and [Dec. No. 40146/14] for the financial support given to this research.

Open Access This article is distributed under the terms of the Creative Commons Attribution 4.0 International License (http://creativeco mmons.org/licenses/by/4.0/), which permits unrestricted use, distribution, and reproduction in any medium, provided you give appropriate credit to the original author(s) and the source, provide a link to the Creative Commons license, and indicate if changes were made.

\section{References}

1. B.E. Conway, Electrochemical Supercapacitors: Scientific Fundamentals and Technological Applications (Springer, Berlin, 2013)

2. F. Béguin, E. Frąckowiak, Supercapacitors: Materials, Systems, and Applications (Wiley, Amsterdam, 2013)

3. J. Rinne, J. Keskinen, P.R. Berger, D. Lupo, M. Valkama, Int. J. Wirel. Inf. Netw. 24, 291 (2017)

4. J. Rinne, J. Keskinen, P. Berger, D. Lupo, M. Valkama, Sensors 18, $3992(2018)$

5. T.M. Kraft, J. Keskinen, M. Pulkkinen, M. Silvennoinen, T. Kankkunen, M. Li, A. Railanmaa, S.S. Chouhan, T. Haapala, K. Halonen, S. Jamalizavareh, J. Salomaa, S. Siitonen, R. Ekqvist, D. Lupo, M. Mäntysalo, in (OE-A Demo Competition, LOPEC 2016, Munich). Accessed 6 April 2016

6. A. Railanmaa, S. Lehtimäki, D. Lupo, Appl. Phys. A 123, 459 (2017)

7. N.A. Choudhury, S. Sampath, A.K. Shukla, Energy Environ. Sci. 2, 55 (2009)

8. A. Al-Kahlout, D. Vieira, C.O. Avellaneda, E.R. Leite, M.A. Aegerter, A. Pawlicka, Ionics (Kiel). 16, 13 (2010)

9. D.F. Vieira, C.O. Avellaneda, A. Pawlicka, Electrochim. Acta 53, 1404 (2007)

10. M.F. Shukur, F.M. Ibrahim, N.A. Majid, R. Ithnin, M.F.Z. Kadir, Phys. Scr. 88, 25601 (2013)

11. R.F.M.S. Marcondes, P.S. D'Agostini, J. Ferreira, E.M. Girotto, A. Pawlicka, D.C. Dragunski, Solid State Ion. 181, 586 (2010)

12. J.R. Andrade, E. Raphael, A. Pawlicka, Electrochim. Acta 54, 6479 (2009)

13. E. Raphael, C.O. Avellaneda, B. Manzolli, A. Pawlicka, Electrochim. Acta 55, 1455 (2010)

14. Y. Xu, Z. Lin, X. Huang, Y. Liu, Y. Huang, X. Duan, ACS Nano 7, 4042 (2013)

15. Y.J. Kang, H. Chung, C.-H. Han, W. Kim, Nanotechnology 23, $065401(2012)$

16. C. Meng, C. Liu, L. Chen, C. Hu, S. Fan, Nano Lett. 10, 4025 (2010)

17. M. Areir, Y. Xu, D. Harrison, J. Fyson, Mater. Sci. Eng. B 226, 29 (2017)

18. S. Lehtimäki, M. Li, J. Salomaa, J. Pörhönen, A. Kalanti, S. Tuukkanen, P. Heljo, K. Halonen, D. Lupo, Int. J. Electr. Power Energy Syst. 58, 42 (2014)

19. T. Kraft, M. Kujala, A. Railanmaa, S. Lehtimäki, T. Kololuoma, J. Keskinen, D. Lupo, M. Mäntysalo, in IEEE Nano (IEEE Xplore, 2018), Cork, Ireland. https://ieeexplore.ieee.org/document/86262 90

20. S. Lehtimäki, A. Railanmaa, J. Keskinen, M. Kujala, S. Tuukkanen, D. Lupo, Sci. Rep. 7, 46001 (2017)

21. IEC 62391-1 (2006). Accessed 25 May 2017

22. J. Keskinen, A. Railanmaa, D. Lupo, J. Energy Storage 16, 243 (2018)

Publisher's Note Springer Nature remains neutral with regard to jurisdictional claims in published maps and institutional affiliations. 